\title{
COLLIMATOR SYSTEMS FOR THE SNS RING
}

\author{
H. Ludewig, N. Simos, J. Walker, P. Thieberger, A. Aronson, J. Wei, M. Todosow, \\ BNL, Upton, NY
}

\begin{abstract}
The requirements and performance goals for the collimators are to reduce the uncontrolled beam loss by $2 \times 10^{-4}$, absorb $2 \mathrm{~kW}$ of deposited heat, and minimize production and leakage of secondary radiation. In order to meet these requirements a self-shielding collimator configuration consisting of a layered structure was designed. The front layers (in the direction of the proton beam) are relatively transparent to the protons, and become progressively less transparent (blacker) with depth into the collimator. In addition, a high density (iron) shield is added around the outside. The protons will be stopped in the center of the collimator, and thus the bulk of the secondary particles are generated at this location. The conceptual design described, the method of analysis discussed, and preliminary performance parameters outlined.
\end{abstract}

\section{INTRODUCTION}

Collimators are used to remove halo or off-momentum particles from the main proton beam. In addition to removing halo particles collimators will also act as shielding for the remainder of the accelerator structures. Thus, collimators reduce uncontrolled losses around the ring and reduce activation of the accelerator components.

Requirements and performance goals for the collimator are summarized below:

1. Halo proton attenuation by a factor of $2 \times 10^{-4}$,

2. Minimize production of secondary radiation, and its subsequent leakage, and

3. Remove heat $(2 \mathrm{~kW})$.

In order to meet these goals a self-shielding collimator configuration will be designed. An arrangement consisting of a layered structure will be considered. The initial layers (in the direction of the proton beam) are transparent to protons, and become progressively less transparent (blacker) with depth into the collimator. In addition, a high density (iron) shield will be added around this structure. The protons are stopped in the approximate center of the collimator, and thus the bulk of the secondary particles will also be generated there. Since these secondary particles are primarily produced isotropically their leakage path length will be maximized in this manner (high probability of capture or attenuation). In the case of neutrons a black layer is included at each end in order to further minimize their leakage in the direction of the beam. This design will therefore minimize the activation of surrounding accelerator components.

\section{CONCEPTUAL DESIGN}

The conceptual design, based on the above requirements and ring constraints, is shown on Figure 1. The protons travel from left to right, with the beam confined primarily to the inner diameter of the collimator. Halo particles are found between the collimator inner diameter and the beam tube inner diameter, and are assumed to pass into the collimator volume. On their way into the collimator the halo particles will first encounter the tapered transition surface between the collimator tube and the vacuum chamber. This surface has a wall thickness of $1 \mathrm{~cm}$ and is made of steel. The next $15 \mathrm{~cm}$ consist of a borated lightwater volume. This region is relatively transparent to high energy protons, but lower energy neutrons would be thermalized in this region and be absorbed by the boron. The use of borated light water to thermalize and absorb neutrons is a common practice in the light water reactor industry.

All the zones to this point have the same composition in the radial direction. The following two zones have a radial variation at a radius of $20 \mathrm{~cm}$. Within the $20 \mathrm{~cm}$ radius they consist of randomly packed spheres cooled by borated light water, and outside this radius they consist of solid iron plates. This arrangement is chosen to ease the assembly of the collimator, ensure heat removal, and minimize the cost. Randomly packed beds of particles are particularly efficient at heat transfer, since their area per unit volume is very large. Furthermore, the cost of small spheres of stainless steel is lower than machined discs of the same material. The void (coolant in this case) fraction of randomly packed spheres is approximately $35 \%$, thus the solid fraction in these zones will be $65 \%$. The $100 \mathrm{~cm}$ long particle bed zone will consist of $3 \mathrm{~mm}$ diameter stainless steel particles. The protons will lose the bulk of their energy in this zone, and since the production of neutrons per proton is modest for stainless steel, the secondary production of neutrons is relatively low. However, there is a probability of generating secondary protons in addition to the neutrons. Fortunately the yield of secondary protons is low compared to the neutron yield, due to the fact that the protons have to overcome the potential barrier before escaping the excited nucleus. 
Finally, the back $15 \mathrm{~cm}$ of the collimator consists of the same borated light water used in the first $15 \mathrm{~cm}$ of the collimator. This volume will ensure that many of the remaining spallation neutrons are slowed down and captured. The collimator is encased in $45 \mathrm{~cm}$ of solid iron on all sides. The collimator thus has an overall radius of $75 \mathrm{~cm}$ and a total length of $222 \mathrm{~cm}$ (including the iron shield).

\section{ANALYSIS AND RESULTS}

The above collimator configuration was analyzed using the Monte Carlo codes LAHET [1], for particles above 20 $\mathrm{MeV}$; and MCNP [2] for particles below $20 \mathrm{MeV}$. In addition, a suitably modified version of the ORIGEN [3] code was used to estimate the buildup of spallation products during machine operation, and their decay following shutdown. The proton beam is assumed to be traveling from left to right, parallel to the collimator tube. The source plane is situated at the transition piece. Radially the proton beam is assumed to have a Gaussian shape.

These results show that the backward (opposite to the direction of the proton beam) and forward (in the direction of the proton beam) proton currents in the halo zone of the beam (radius greater than $5 \mathrm{~cm}$ ) decrease monotonically to the back end of the collimator. In addition, the leakage out of the front end of the collimator is also found to be vanishingly small. Within the collimator the proton current in the backward direction varies, with a maximum at the interface between the shield and the collimator containment vessel. The need for the thick iron shield is thus demonstrated. Thus, the proton leakage out of the back and front of the collimator meets the design goal set for it. Neutron currents (neutrons with energies above 20 $\mathrm{MeV}$ ) were also determined. It was seen that in the forward direction the neutron current increases initially and then decreases monotonically to a low value at the outer surface. In the backwards direction the current peaks at the interface between the front shield and the collimator body.

The thick shield should minimize neutron leakage, which in turn will minimize the activation of the tunnel air. The only other activated material which can leave the collimator is the cooling water. Potentially ${ }^{7} \mathrm{Be}$ and ${ }^{3} \mathrm{H}$ are formed, and circulate in the coolant. For the above reason the cooling water will be cooled in a closed loop via an intermediate heat exchanger. The maximum heat load from a collimator is $2 \mathrm{~kW}$. If a temperature rise of $5^{\circ} \mathrm{C}$ is assumed $\left(T_{n}=30^{\circ} \mathrm{C}\right.$, and $\left.T_{\text {out }}=35^{\circ} \mathrm{C}\right)$ a flow rate of approximately $3 \times 10^{4} \mathrm{~m}^{3} / \mathrm{s}$ is required. This implies a moderate heat removal system for the design basis condition.
Estimates of the energy deposition in the collimator indicate the bulk of the power will be generated in the inner $20 \mathrm{~cm}$ of the front borated light water zone $(9 \%)$, the front iron shield (10\%), and the stainless steel particle bed $(70 \%)$. All these zones need to be cooled by the cooling water system. The stainless steel particle bed zone is inside the collimator and is cooled by the borated water system. Preliminary estimates of the temperature indicate that they are well within the operating limits.

In addition to the estimates of energy deposition in bulk components, an estimate of the axial and radial variation of energy deposition was made in the collimator tube, and front shell. The energy deposition was found to be quite modest, with the maximum $\left(2.2 \times 10^{5} \mathrm{~W} / \mathrm{m}^{3}\right)$ occurring at the leading edge. The tube and containment shell are subject to the most challenging thermal environment, since they are cooled on one surface, and the coolant flow pattern in the leading edge comer can be ambiguous. Assuming a heat transfer coefficient of $100 \mathrm{~W} / \mathrm{m}^{2}-{ }^{\circ} \mathrm{C}$ on the surface and a coolant bulk temperature of $32^{\circ} \mathrm{C}$ the maximum temperature rise in the stainless steel collimator tube is approximately $12^{\circ} \mathrm{C}$. The associated thermal stress is $9 \times 10^{6} \mathrm{~N} / \mathrm{m}^{2}$. These values are well within the operating limits of the containment shell material. The cyclic nature of the beam appears to have very minimal impact on the thermal response of the shell. However, it should be pointed out that possible thermal fatigue effects from the cyclic beam off and on condition as well as from thermalmechanical shock have not been addressed yet.

It was assumed that the machine has operated for 180 days at full power ( $1 \mathrm{MW}$, with 0.001 of the beam being captured in the collimator). Activation levels after 1 day, 7 days, and 30 days following shutdown were determined. It was found that the quadrupole magnets have an activation of 5 Curies. The primary activation products being ${ }^{51} \mathrm{Cr},{ }^{54} \mathrm{Mn},{ }^{56} \mathrm{Mn},{ }^{55} \mathrm{Fe},{ }^{59} \mathrm{Fe},{ }^{65} \mathrm{Ni},{ }^{62} \mathrm{Cu}$, and ${ }^{66} \mathrm{Cu}$. In the iron zones of the magnet structure the same activation products are important, except $\mathrm{Cu}$ and $\mathrm{Ni}$. The magnets behind the collimator have a vanishingly small amount of radioactive buildup. The energy spectrum due to decay gamma rays was found to peak in the energy range between $0.85 \mathrm{MeV}$ and $1.25 \mathrm{MeV}$. The activity of the solid components within the collimator is well shielded.

Furthermore, the question of scraping interactions, on the collimator walls of primary protons and the production of secondary particles is being investigated. A simulation of primary proton scrapping on the walls of a conical shaped collimator with an inner diameter of $10 \mathrm{~cm}$ at its entrance, for various included conical angles, and a length of $1 \mathrm{~m}$, was carried out. The results indicate that for a scraping angle of $0.05^{\circ}-50 \%$ of the particles re-emerge from the collimator, and $47 \%$ are confined to a cone with an included angle of $30^{\circ}$. This fraction decreases 
monotonically with increasing included collimator angle $\left(\sim 40 \%\right.$ for $\left.0.2^{\circ}\right)$. The energy distribution of the emerging protons has a peak at the primary proton energy, but has a low energy tail which extends down to $100 \mathrm{McV}$. The production of secondary electrons at SNS conditions has been estimated. First, based on a theory due to Sternglass $[4,5]$, and second scaled from experiments using the above theory [6]. The values of electron production per primary proton ( $\left(\mathrm{e}^{\mathrm{T}} \mathrm{p}^{+}\right)$vary from 200 to 25 for the above two estimates respectively. Currently, an experimental program is being carried out at BNL to study this phenomena. The objective of these experiments is to further quantify the processes involved, and more Importantly to investigate possible methods of suppressing the production/capture of secondary electrons.

Finally, the possibility of incorporating a movable inner surface to the primary ring collimator is being investigated. A possible configuration is shown on Figure 2. The inner wall is constructed in halves which move in and out radially both vertically and horizontally, to vary the aperture. The pieces are made of copper with internal cooling loops. Initial estimates indicate that the energy Jeposition and neutron production is $10 \%-15 \%$ higher than if a similar piece was made of stainless steel. However, due to its superior heat conduction, copper will se used in this application.

\section{ACKNOWLEDGMENTS}

This work was performed under the auspices of the U. S. Jepartment of Energy. Research on the SNS is sponsored gy the Division of Material Sciences, U. S. Department of Energy, under contract number DE-AC05960R22464 vith Lockheed Martin Energy Research Corporation for Jak Ridge National Laboratory.

\section{REFERENCES}

I R.E. Prael and H. Lichtenstein, "User Guide to LCS: The LAHET Code System," Los Alamos National Laboratory, Los Alamos, NM, LA-UR-89-3014 (1989)

-1 MCNP-A General Monte Carlo N-Particle Transport Code Version 4A, J.F. Breismeister, ed., Los Alamos National Laboratory, Los Alamos, NM, LA-12625-M (1993).

¿) A.G. Croff, "ORIGEN2 - A Revised and Updated Version of the Oak Ridge Isotope Generation and Depletion Code," Oak Ridge National Laboratory, Oak Ridge, TN, ORNL-5621 (1980).

.1 E.J. Sternglass, Phys, Rev. 108, \#1,1 (1957).

$\therefore \quad$ M. Plum, PSR-95-001, LANL, (1995).

․ S.Y. Zhang, "Secondary Electron Emission at the SNS Storage Ring Collimator," SNS Tech Note \#50 (1998).

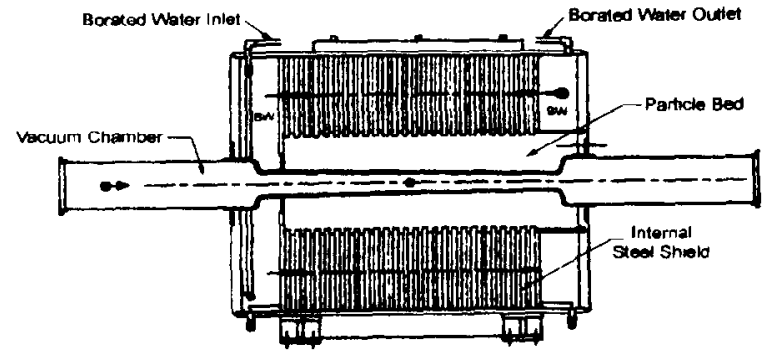

Figure 1: Schematic of Beam Collimator

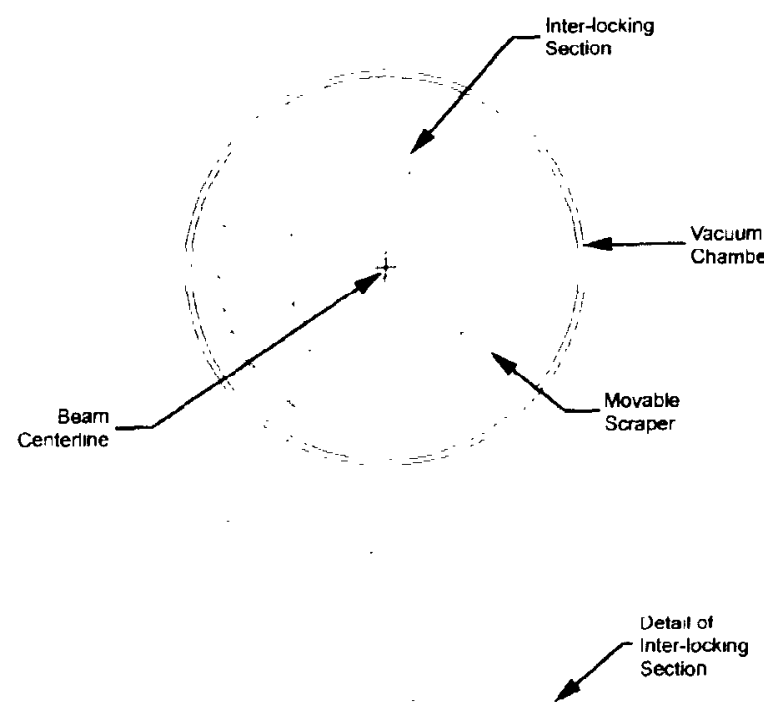

Figure 2: Moveable Beam Scrapers 